\title{
Key-Skeleton Based Feedback Tool for Assisting Physical Activity
}

\author{
Renato Baptista $^{1}$, Enjie Ghorbel ${ }^{1}$, Abd El Rahman Shabayek ${ }^{1,2}$, Djamila Aouada ${ }^{1}$, Björn Ottersten ${ }^{1}$ \\ ${ }^{1}$ Interdisciplinary Centre for Security, Reliability and Trust (SnT), University of Luxembourg \\ 2 Computer Science department, Faculty of Computers and Informatics, Suez Canal University, Egypt \\ \{renato.baptista, enjie.ghorbel, abdelrahman.shabayek, djamila.aouada, bjorn.ottersten\}@uni.lu
}

\begin{abstract}
This paper presents an intuitive feedback tool able to implicitly guide motion with respect to a reference movement. Such a tool is important in multiple applications requiring assisting physical activities as in sports or rehabilitation. Our proposed approach is based on detecting key skeleton frames from a reference sequence of skeletons. The feedback is based on the 3D geometry analysis of the skeletons by taking into account the key-skeletons. Finally, the feedback is illustrated by a colorcoded tool, which reflects the motion accuracy.

Index Terms-Key-skeleton, Feedback, Stroke
\end{abstract}

\section{INTRODUCTION}

Physical activity is essential for maintaining a healthy lifestyle. For stroke survivors, exercising is crucial in order to recover some autonomy in their daily life activities [1]. Nevertheless, many stroke survivors do not exercise regularly due to several reasons, such as fatigue, lack of motivation, confidence and skill levels [2]. Generally, stroke survivors undergo physical therapy supervised by a healthcare professional, who usually suggests activities for a home-based rehabilitation [3]. Therefore, having an automatic and easily accessible feedback system at home motivates the patients to do more exercises.

Over the last decade, the availability of RGB-D sensors, which are able to detect humans and approximate a 3D skeleton in real-time has boosted the utilization of feedback tools for assisting physical activities [4], [5]. In this paper, we propose an intuitive feedback tool that combines [4] and [5]. This work is organized as follows: in Section II, the details of the feedback proposals with respect to a reference movement are described. System deployment is presented in Section III, and finally, Section IV concludes the paper.

\section{FEedBack Proposals}

In order to support physical activity while exercising at home, the authors of [4] proposed a framework that provides a visual feedback through human interpretable messages. In [4], the feedback proposals are computed by comparing the movement with a template skeleton pose, without specifying pose constraints of joint configurations. On the other hand, Baptista et al. [5] presented a system for posture monitoring and correction. In their work, they provide visual feedback to the user in order to correct postural defects, specially for the body-part involving the back of the subject. To design a more complete feedback tool, we propose to combine and adapt the two approaches presented in [4] and [5]. Consequently, the feedback is proposed with respect to key-skeletons, which are defined as the skeleton sequence that preserve the flow of a given movement.

While exercising at home, post-stroke survivors have difficulty on performing specific movements such as reaching determined height with the spastic arm. In order to complete the movement, most of the time, patients tend to use movement compensation. Movement compensation refers to the motion produced by other body-parts to compensate the lack of mobility of the damaged body-part. In most of the cases, patients tend to use their back to support the proposed exercise. Having such a system that combines feedback proposals and posture monitoring, the problem of movement compensation can be addressed by analyzing the deviation of the posture from a correct posture. In summary, the contributions of this work are: 1) the selection of key-skeleton frames that preserve the flow of the reference movement; 2) the feedback proposals which are implicitly shown while exercising using a colorcoded illustration; and 3) the coupling of body movement compensation feedback and guiding movement feedback.

In this section, we describe how the feedback is presented in the form of color-coded information, with the objective of helping the user to correct the current movement.

Let $\mathrm{S}=\left\{\mathbf{j}_{1}, \cdots, \mathbf{j}_{N}\right\}$ be a skeleton of a human subject with $N$ joints. Each joint is defined by its 3D coordinates $\mathbf{j}=\left[j_{x}, j_{y}, j_{z}\right]^{\top}$. A movement is defined as a skeleton sequence $\mathrm{M}=\left\{\mathrm{S}_{1}, \cdots, \mathrm{S}_{k}\right\}$, where $k$ is the number of skeletons of the sequence. In this work, the goal is to provide feedback to a movement being performed by comparing it to a reference movement $\hat{M}$. Thus, in order to handle speed variations, a temporal alignment technique is usually applied, e.g., Dynamic Time Warping (DTW) [6]. Contrary to [7], to provide feedback for the current skeleton, we do not apply any temporal alignment technique between the movement being performed and the reference movement. Instead, we uniformly sample the reference movement $\hat{M}$ into a fixed number of skeletons $q$, denoted as key-skeletons, represented by $\phi$. This process is chosen because the usage of temporal alignment techniques, e.g., DTW, is not possible since the feedback should be provided in real-time. Indeed, these techniques require the complete knowledge of both sequences. Thus, the key-skeletons preserve the temporal information and allow the real-time transmission of the feedback proposals to the user. Fig. 1 shows an example of the key-skeletons extraction, where 
the highlighted skeletons in green illustrate the key-skeletons.

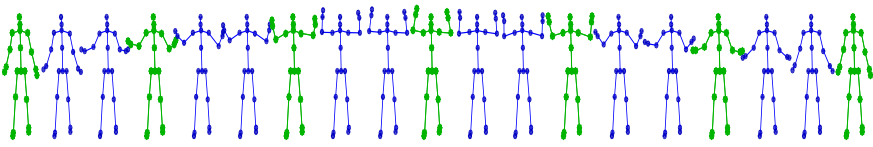

Fig. 1: Key-skeleton extraction from a reference movement. The green colored skeletons represent the key-skeletons.

While comparing two skeleton sequences, the first step is to spatially register each skeleton. This is done in order to ensure invariance to absolute locations and scale. For that purpose, we normalize each skeleton following the work of [4], [5]. Similar to [5], we represent a human skeleton as a set of body-parts, where each body-part is composed of a set of specific joints. Considering this, we adopt the metric of [4] to compute the error $\xi(\cdot, \cdot)$ between the static current skeleton pose and the key-skeleton pose. This is done by computing the transformation that each body-part needs to undergo for decreasing a score function [4].

Thus, given a skeleton stream $M$, the feedback for the current skeleton $M_{c}$ is presented with respect to the keyskeleton $\hat{\mathrm{M}}_{\phi}^{i} \forall i \in[1, \cdots, q]$ such that

$$
i=\left\{\begin{array}{ll}
i+1 & , \text { if } \xi\left(\mathrm{M}_{c}, \hat{\mathrm{M}}_{\phi}^{i}\right)<\delta \\
i & , \text { otherwise }
\end{array},\right.
$$

where $\delta$ is a predefined threshold that allows the sequential iteration of the key-skeletons while preserving the flow of the movement. In addition, the current error $\xi\left(\mathrm{M}_{c}, \hat{\mathrm{M}}_{\phi}^{i}\right)$ is translated into a color code in order to be shown to the user. Thus, when $\xi\left(\mathrm{M}_{c}, \hat{\mathrm{M}}_{\phi}^{i}\right)$ is close to $\delta$, green is the color that is going to be shown. Subsequently, when $\xi\left(\mathrm{M}_{c}, \hat{\mathrm{M}}_{\phi}^{i}\right)$ is far from $\delta$, red is the color to be shown.

\section{System DePloyment}

In this section, we present the proposed color-coded tool in order to implicitly provide feedback to the user while performing a movement.

We tested our system with healthy subjects and patients using the following two step scenario: 1) the exercise of interest is shown to the user; and 2) the user starts exercising following the provided feedback (color-coded tool) only. Fig. 2 shows the combined feedback proposals of one of the patients. While Fig. 2a demonstrates a correct arm movement coded in green after being compared to the reference movement, Fig. $2 \mathrm{~b}$ demonstrates an incorrect position of the arm coded in orange. Finally, Fig. 2c shows color-feedback proposals to restrict the body compensation movement. This compensation is not desirable since the patient is using other body-parts to help on the performance of the exercise which may lead to long-term injuries. Globally, the ideal situation is to keep the back and the trained body-part colored in green while performing the exercise (see Fig. 2d).

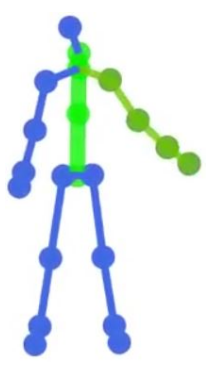

(a)

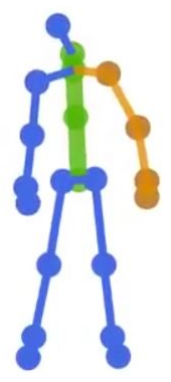

(b)

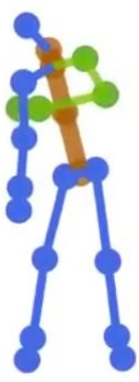

(c)

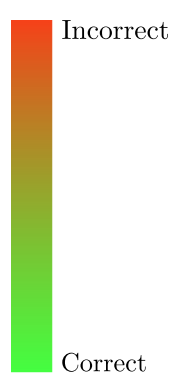

(d)
Fig. 2: Combined feedback proposals. Fig. 2a and $2 b$ show the example where the position of the arm goes from correct to incorrect, respectively. Fig. 2c illustrates the case of movement compensation with the back of the user. Fig. $2 d$ depicts the color bar of the feedback proposals.

\section{CONCLUSION}

In this paper, we presented a tool to implicitly provide feedback to the user with the objective of improving the movement being performed with respect to a reference movement. Preliminary results with real patients showed the potential of the color-coded tool to guide patients towards a given reference movement.

\section{ACKNOWLEDGMENT}

This work has been funded by the European Union's Horizon 2020 research and innovation project STARR under grant agreement No.689947. We thank Julien Pager, Alice Douchet, Mathilde André and Stéphane Bouilland from HOPALE, France, for giving useful information about the patients' preferences. We also thank Sabrina Panëels, Margarita Anastassova and Mehdi Boukallel from CEA, France, for giving constructive feedback. And finally, we are particularly grateful to the patients that participated in the tests and had given their data.

\section{REFERENCES}

[1] G. Kwakkel, B. J. Kollen, and H. I. Krebs. Effects of robot-assisted therapy on upper limb recovery after stroke: a systematic review. Neurorehabilitation and Neural Repair, 2007.

[2] F. Ofli, G. Kurillo, S. Obdrzalek, R. Bajcsy, H. B. Jimison, and M. Pavel. Design and evaluation of an interactive exercise coaching system for older adults: Lessons learned. IEEE J. Biomedical and Health Informatics, 2016

[3] P. Langhorne, G. Taylor, G. Murray, M. Dennis, C. Anderson, E. BautzHolter, P. Dey, B. Indredavik, N. Mayo, M. Power, et al. Early supported discharge services for stroke patients: a meta-analysis of individual patients data. The Lancet, 2005.

[4] M. Antunes, R. Baptista, G. Demisse, D. Aouada, and B. Ottersten. Visual and human-interpretable feedback for assisting physical activity. In European Conference on Computer Vision (ECCV) Workshop on Assistive Computer Vision and Robotics Amsterdam, 2016.

[5] R. Baptista, M. Antunes, A. E. R. Shabayek, D. Aouada, and B. Ottersten. Flexible feedback system for posture monitoring and correction. In IEEE International Conference on Image Information Processing (ICIIP), 2017.

[6] M. Müller. Dynamic Time Warping. Springer, 2007.

[7] R. Baptista, M. Antunes, D. Aouada, and B. Ottersten. Video-Based Feedback for Assisting Physical Activity. In International Joint Conference on Computer Vision, Imaging and Computer Graphics Theory and Applications (VISAPP), 2017. 